\title{
Sensibilisierungstrainings gegenüber häuslicher Gewalt für medizinisches Fachpersonal. Sind diese auch effizient?
}

\author{
Thomas Beck • Iris Trawöger · David Riedl · Astrid Lampe
}

Eingegangen: 29. Juli 2019 / Angenommen: 11. Februar 2020 / Online publiziert: 28. Februar 2020

(c) Der/die Autor(en) 2020

\section{Zusammenfassung}

Hintergrund Untersuchungen der Europäischen Union folgend, sind $33 \%$ aller Frauen ab dem 15. Lebensjahr von häuslicher Gewalt betroffen und wenden sich bei der Suche nach Hilfe vorwiegend an das Gesundheitssystem.

Das Erleben von Gewalt hat schwerwiegende Folgen nicht nur auf die psychische, sondern auch auf die körperliche Gesundheit der Betroffenen. So kommt es neben Verletzungsfolgen, u.a. zu unerklärbaren Schmerzen, gastrointestinalen Beschwerden oder Problemen im Bereich der reproduktiven Medizin.

Nachdem sich viele der Betroffenen auf der Suche nach Unterstützung zuerst an das Gesundheitssystem wenden, kommt dem medizinischen Fachpersonal auch hier eine Schlüsselrolle zu, wodurch Sensibilisierungstrainings für die Berufsgruppen immer bedeutender werden.

Methode Teilnehmerinnen und Teilnehmer an Sensibilisierungstrainings gegenüber häuslicher Gewalt wurden vor und nach dem Training mit Hilfe eines Fragebogens $\mathrm{zu}$ ihrer empfundenen Sicherheit im Umgang mit von Gewalt betroffenen Patientinnen und Patienten befragt.

Ergebnisse Die Evaluation eines Schulungszyklus am Landeskrankenhaus Innsbruck zeigte signifikante Verbesserungen im subjektiven Sicherheitsgefühl im Umgang mit gewaltbetroffenen Patientinnen und Patienten.

PD Dr. T. Beck $(\bowtie) \cdot$ I. Trawöger · D. Riedl · A. Lampe

Universitätsklinik für Medizinische Psychologie,

Medizinische Universität Innsbruck,

Speckbacherstraße 23, 6020 Innsbruck, Österreich

thomas.beck@tirol-kliniken.at

PD Dr. T. Beck · I. Trawöger · A. Lampe

Opferschutzgruppe des Landeskrankenhauses Innsbruck,

Anichstraße 35, 6020 Innsbruck, Österreich
Fazit für die Praxis Ein strukturierter Aufbau der Sensibilisierungstrainings mit Basiskursen und Aufbauseminaren, sowie einem Fokus auf Beziehungsaufbau und Umgang mit Emotionen erscheint sinnvoll.

Schlüsselwörter Häusliche Gewalt · Medizinisches Fachpersonal · Sensibilisierungstrainings · Beziehungsaufbau

Awareness training for domestic violence for medical professionals. Are they efficient too?

\section{Summary}

Background According to studies conducted by the European Union, 33\% of all women over the age of 15 are affected by domestic violence and tend to turn to the health care system to seek help.

The experience of violence has serious consequences not only for the psychological, but also for the physical health of those affected. Except direct injury consequences, inexplicable pain, gastrointestinal discomfort or problems in the field of reproductive medicine can be observed, for example.

With many of those affected turning to the health care system first in search of support, healthcare professionals also have a key role to play here, making sensitization training for professional groups increasingly important.

Method Participants in awareness-raising training on domestic violence were interviewed before and after training with a questionnaire on their perceived safety in dealing with patients affected by violence.

Results The evaluation of a sensitization training at the Landeskrankenhaus Innsbruck showed significant improvements in the subjective sense of security in dealing with patients affected by violence.

Conclusion for practice A clear structure of sensitization trainings with basic courses and advanced train- 
ing seminars, as well as a focus on developing relationships and dealing with emotions, makes sense.

Keywords Domestic violence - Medical staff • Building of relationship

\section{Hintergrund}

Häusliche Gewalt ist ein in Europa weitverbreitetes Problem. So berichtet die Agentur der Europäischen Union für Grundrechte, dass innerhalb der EU etwa $33 \%$ aller Frauen seit ihrem 15. Lebensjahr von ihrem Partner bzw. ihrer Partnerin oder einer anderen im Haushalt lebenden Person körperliche und/oder sexuelle Gewalt erfahren haben. In Deutschland sind $35 \%$, in Österreich $20 \%$ [1] der Frauen betroffen. Eine bevölkerungsbasierte Studie in Österreich zeigt, dass Frauen im Laufe ihres Lebens zu 30\% körperlicher Gewalt, $40 \%$ psychischer und $30 \%$ sexueller Gewalt ausgesetzt sind. Für Männer liegen die Werte bei $28 \%$ für körperliche, $31 \%$ für psychische und 8,8\% für sexuelle Gewalt [2]. Frauen, die in Notaufnahmen aufgenommen werden, erleben aktuell häusliche Gewalt, mit einer Lebenszeitprävalenz von 36,6\% [3].

Das Erleben von Partnergewalt (IPV) und sexualisierter Gewalt (SV) sind bedeutende Gesundheitsrisiken für Frauen und ihre Kinder [4]. Die mit Gewalt in Verbindung stehenden gesundheitlichen Folgen sind vielfältig: Somatische und psychosomatische Symptome (z.B. Prellungen, Frakturen, Gesichtsund Schädelverletzungen, unerklärliche Schmerzen, gastrointestinale Beschwerden sowie Herz-KreislaufSymptome), psychische Erkrankungen (Belastungsund Stressreaktionen, Essstörungen, Schlafstörungen, Angst- und Panikerkrankungen, PTSD, Depression und Suizidalität) sowie gynäkologische Erkrankungen (Fehl- und Frühgeburten, Schwierigkeiten während Schwangerschaft und Geburt, geringes Geburtsgewicht) und Entwicklung einer Suchterkrankung (Rauchen, Alkohol- und Drogengebrauch [5, 6]). Auch das Erleben von Gewalt während der Kindheit und Jugend kann in der Folge zur Ausbildung körperlicher Erkrankungen, wie beispielsweise Herzkreislauferkrankungen, Diabetes oder Schlaganfällen, führen sowie ein deutliches erhöhtes Risikoverhalten fördern [7]. In einer aktuellen Studie konnte gezeigt werden, dass die Kombination von frühkindlichen Traumati- sierungen und häuslicher Gewalt im Erwachsenenalter die Auftretenswahrscheinlichkeit verschiedener körperlicher Erkrankungen (chronische Schmerzen, gastrointestinale Erkrankungen, Atemwegserkrankungen, Stoffwechselstörungen) signifikant erhöhen [8].

Gewaltbetroffene nehmen häufiger ärztliche Hilfe in Anspruch als Frauen, die keiner Gewalt ausgesetzt sind. $60 \%$ der Frauen, die Gewalt durch ihren Partner erleben, suchen Hilfe im Gesundheitssystem $(23 \%$ in Krankenhäusern), wo hingegen nur $29 \%$ der Betroffenen Hilfe in Opferschutzorganisationen wie Frauenhäusern oder anderen sozialen Einrichtungen suchen [1]. Aus den Ergebnissen unser kürzlich publizierten eigenen Studie wissen wir, dass am Landeskrankenhaus Innsbruck auch 15,7\% der befragten Männer angegeben haben, von Gewalt betroffen zu sein [9].

Zumeist berichten die Frauen nicht spontan von der erlebten Gewalt. Sie schämen sich, denken, sie seien selber Schuld oder ein Erzählen würde ihre Situation nur verschlimmern. Es ist einerseits die Angst vor dem Täter (z.B. „wenn du jemanden etwas erzählst, bring ich dich um"), aber auch die Angst vor den Behörden (z. B. Wegnahme der Kinder von Seiten des Jugendamts), die ein spontanes Berichten und das aktive Aufsuchen von Hilfe von Seiten der Betroffenen verhindert.

Somit kommt dem medizinischen Fachpersonal eine bedeutende Rolle bei der Identifikation und Behandlung von gewaltbetroffenen Personen zu [10, 11]. Dennoch werden in Krankenhäusern noch immer deutlich zu wenig von Gewalt betroffene Menschen vom medizinischen Fachpersonal erkannt und auf ihre Situation angesprochen $[12,13]$.

Von Seiten des medizinischen Fachpersonals werden vor allem ein Mangel an Zeit, die abweisende Haltung der Patientinnen und Patienten und auch „ein Vergessen“, danach zu fragen (vielleicht ein Produkt der gemeinsamen Abwehr der Patientinnen und der Behandler), als Hindernisse genannt [14]. Auch eine spürbare Unsicherheit von Behandlerinnen und Behandlern nach Gewalt zu fragen, kann es den Betroffenen erschweren, Gewalterfahrungen zu thematisieren [15]. Hinzu kommt die Tatsache, dass Personen jeden Alters, sozialen Status und ethnischer Zugehörigkeit von Gewalt betroffen sein können, so dass es außer dem Auftreten spezieller Verdachtsmomente im Kon-
Tab. 1 Sensibilisierungsschulungen am Landeskrankenhaus Innsbruck

\begin{tabular}{|l|l|}
\hline Modul 1 (Dauer: $15 \mathrm{~min})$ & $\begin{array}{l}\text { Informationen über die OSG (Ziele, Aufgaben, Angebote) } \\
\text { Vorstellung der Mitglieder } \\
\text { Vorstellung Patientenpfad }\end{array}$ \\
\hline Modul 2 (Dauer: $90 \mathrm{~min}$ ) & $\begin{array}{l}\text { Spezifische Informationen über häusliche Gewalt } \\
\text { Hinweise zur Gesprächsführung } \\
\text { Typische Verletzungsmuster } \\
\text { Falldiskussionen möglich }\end{array}$ \\
\hline Modul 3 (Dauer: $1 \mathrm{Tag}$ ) & $\begin{array}{l}\text { Erkennung Betroffener } \\
\text { Kommunikation mit Betroffenen (Information und Training) } \\
\text { Typische Verletzungsmuster und Dokumentation } \\
\text { Rollenspiele } \\
\text { Falldiskussionen }\end{array}$ \\
\hline
\end{tabular}


takt keine spezifischen Merkmale gibt, die auf Gewalt hinweisen [16].

In Studien wurde immer der Nutzen eines generellen Screenings nach Gewalt in Krankenhäusern diskutiert [17, 18]. Ein Screening nach Gewalterfahrung kann neben der Entlastung des Personals den zusätzlichen Effekt haben, dass Betroffenen das Problem bewusster werden kann, auch wenn es ihnen zu diesem Zeitpunkt vielleicht noch nicht möglich ist, Veränderungen oder Handlungen zu setzen [19].

Die frühe Identifikation und adäquate Versorgung von Gewalt-Betroffenen ist eine zentrale Aufgabe des Gesundheitssystems. Dem wurde im Jahr 2011 Rechnung getragen, als von der Republik Österreich ein Gesetz verabschiedet wurde, das Krankenanstalten die Einrichtung einer Opferschutzgruppe (OSG) vorschreibt, denen die Früherkennung häuslicher Gewalt sowie die Sensibilisierung des medizinischen Fachpersonals für häusliche Gewalt obliegt [20]. Die Gründung der OSG im Landeskrankenhaus Innsbruck (LKI) erfolgte im März 2012. Mittlerweile konnte von der OSG des LKI ein dreistufiges Ausbildungsprogramm für medizinisches Fachpersonal implementiert und der erste Zyklus der Ausbildung evaluiert werden. Die Module sind aufeinander aufbauend (von einer Dauer von $15 \mathrm{~min}$, über $90 \mathrm{~min}$ bis $\mathrm{zu}$ einem Tag) und umfassen neben der Vorstellung der OSG, spezifischen Informationen über häusliche Gewalt und Hinweisen zur Gesprächsführung auch Rollenspiele und Falldiskussionen (Details siehe Tab. 1).

\section{Methode}

Seit Beginn der interdisziplinären Schulungen haben mittlerweile rund 1000 Krankenhaus-MitarbeiterInnen an dem mehrstufigen Ausbildungsprogramm teilgenommen. Ca. 1000 TeilnehmerInnen besuchten Modul 1 und 2, ca. 100 absolvierten Modul 3. Nur einige wenige Teilnehmerinnen und Teilnehmer absolvierten vor dem Besuch des Moduls 3 entweder Modul 1 oder 2. Diese wurden nicht in diese Erhebung miteingeschlossen. 76 der Teilnehmerinnen und Teilnehmern von Modul 3 wurde vor und nach dem Training ein Evaluationsbogen ausgehändigt, in dem neben demografischen Fragen (Alter, Geschlecht, Berufsgruppe und Berufserfahrung) auch insgesamt 13 Fragen zur empfundenen Sicherheit im Umgang mit gewaltbetroffenen Patientinnen und Patienten gestellt wurden. Die Daten der vorliegenden Untersuchung wurden in den Jahren 2018 und 2019 erhoben. Dabei wurde eine adaptierte und den in Innsbruck vorliegenden Gegebenheiten angepasste Form der Evaluation gewählt, die bereits bei einem ähnlichen Projekt in Düsseldorf zum Einsatz gekommen ist [21].

Die statistischen Analysen wurden mit SPSS (v22.0) durchgeführt. Die Größe der Effektstärken wurde mittels Cohens' d errechnet, wobei Werte von $d=0,3$ wurden als kleine Effekte, $d=0,5$ als mittelgradige Effekte und $\mathrm{d}=0,8$ als große Effekte interpretiert wurden [22, 23].

\section{Ergebnisse}

Insgesamt konnten 70 Fragebögen in die Auswertung aufgenommen werden. Davon wurden 55 (79\%) von Frauen und 15 (21\%) von Männern ausgefüllt. Im Durchschnitt waren die teilnehmenden Personen 40,5 Jahre alt und seit 5,8 Jahren in ihren Berufen tätig. Es nahmen 35 VertreterInnen der Pflege (50,0\%), 17 SozialarbeiterInnen $(24,3 \%)$ sowie 12 Ärztinnen bzw. Ärzte $(17,1 \%)$ an dem Fortbildungszyklus teil. Weitere 6 Teilnehmerinnen (8,6\%) kamen aus anderen Berufen.

Die Auswertung der Fragebogenuntersuchung hinsichtlich den Veränderungen im Gefühl der Sicherheit im Umgang mit Patientinnen und Patienten mit Gewalterfahrungen nach der Schulung zeigten eine deutliche Verbesserung in den meisten erfragten Bereichen. Bei 11 der dreizehn gestellten Fragen konnten nach der Schulung signifikant bessere Werte in der empfundenen Sicherheit der Teilnehmerinnen und Teilnehmer in Bezug auf gewaltbetroffene Patientinnen bzw. ein signifikant geringeres Stresserleben nachgewiesen werden.

Die deutlichsten Veränderungen (Effektstärke $>0,8$ ) zeigten sich hinsichtlich der Sicherheit im Umgang $(p<0,001 ; \mathrm{d}=1,2)$, der Gesprächsführung mit Gewaltbetroffenen $(p<0,001 ; \mathrm{d}=1,2)$, dem Wissen wie am besten psychologische Hilfe angeboten werden kann $(p<0,001 ; \mathrm{d}=1,2)$ sowie der Kenntnis über Ressourcen, auf die betroffene Patientinnen und Patienten zurückgreifen können ( $p<0,001 ; \mathrm{d}=1,1)$. Lediglich in Bezug auf die Gefahr, sich mit gewaltbetroffenen Patientinnen und Patienten identifizieren zu können sowie der Angst, diese zu retraumatisieren, zeigten sich keine signifikanten Veränderungen durch die Schulung. In diesen Bereichen zeigten die Teilnehmerinnen und Teilnehmer jedoch bereits vor der Schulung recht gute Werte (Details siehe Tab. 2).

\section{Fazit für die Praxis}

Für Betroffene von Gewalt ist das Gesundheitswesen eine der wichtigsten Anlaufstellen auf der Suche nach Hilfe und Unterstützung [1]. Umso bedeutender ist es, dass die Angehörigen der medizinischen Fachberufe sensibel gegenüber dem Thema „Gewalt“ und auch entsprechend sicher darin sind, dies mit den betroffenen Patientinnen und Patienten ansprechen bzw. diese an geeignete Hilfseinrichtungen weitervermitteln zu können.

Das stufenweise Trainingsprogramm, das am Landeskrankenhaus Innsbruck angeboten wird, verbessert das subjektive Gefühl der Sicherheit des medizinischen Fachpersonals im Umgang mit gewaltbetroffenen Patientinnen und Patienten. Am Landeskrankenhaus Innsbruck werden alle drei Module angeboten, 


\section{Tab. 2 Ergebnisse der Prä-, Postbefragung}

\begin{tabular}{|c|c|c|c|c|}
\hline Frage & Mprä & Mpost & $p$ & d \\
\hline Wie sicher fühlen Sie sich im Umgang mit PatientInnen, die Gewalterfahrungen erlebt haben? ${ }^{\mathrm{a}}$ & 5,0 & 7,3 & $<0,001$ & 1,2 \\
\hline $\begin{array}{l}\text { Wie sicher fühlen Sie sich während des Gesprächs, wenn Sie den Verdacht haben, dass eine Ge- } \\
\text { walterfahrung bestehen könnte? }^{\mathrm{a}}\end{array}$ & 5,0 & 7,3 & $<0,001$ & 1,2 \\
\hline Ich kenne Ressourcen, auf die die Patientin/der Patient zurückgreifen kannª & 5,4 & 7,8 & $<0,001$ & 1,1 \\
\hline Ich weiß, wie man Gewaltbetroffenen psychologische Unterstützung anbieten kann ${ }^{\mathrm{a}}$ & 4,6 & 7,3 & $<0,001$ & 1,2 \\
\hline Die Behandlung von Gewaltbetroffenen ist für mich sehr stressiga & 5,2 & 4,3 & 0,031 & $-0,4$ \\
\hline Ich fühle mich unbeholfen bei der Behandlung von PatientInnen mit Gewalterfahrungen ${ }^{\mathrm{a}}$ & 4,5 & 3,6 & 0,002 & $-0,6$ \\
\hline Ich neige dazu, mich mit Gewaltbetroffenen zu identifizieren ${ }^{\mathrm{a}}$ & 2,7 & 2,7 & Nicht sign & \\
\hline Wie sicher fühlen Sie sich, PatientInnen nach Gewalterfahrungen zu fragen? ${ }^{c}$ & 2,5 & 3,0 & $<0,001$ & 0,8 \\
\hline $\begin{array}{l}\text { Wie sicher fühlen Sie sich, auf Berichte von Gewalterfahrungen Ihrer PatientInnen angemessen } \\
\text { reagieren zu können? }\end{array}$ & 2,5 & 3,0 & $<0,001$ & 0,8 \\
\hline Wie sicher fühlen Sie sich, PatientInnen mit Gewalterfahrungen helfen zu können? ${ }^{c}$ & 2,1 & 3,0 & $<0,001$ & 1,4 \\
\hline $\begin{array}{l}\text { Ich fühle mich sicher, Patientlnnen mit Gewalterfahrungen (z. B. an spezialisierte Einrichtungen) } \\
\text { weitervermitteln zu können }\end{array}$ & 2,7 & 3,2 & $<0,001$ & 0,7 \\
\hline Ich fühle mich unwohl, wenn ich PatientInnen nach Gewalterfahrungen fragen solld & 2,8 & 2,2 & $<0,001$ & $-0,7$ \\
\hline $\begin{array}{l}\text { Ich habe Bedenken, dass das Erfragen von Gewalterfahrungen die PatientInnen erneut traumatisie- } \\
\text { ren könnte }^{\mathrm{d}}\end{array}$ & 2,6 & 2,3 & Nicht sign & \\
\hline \multicolumn{5}{|c|}{ 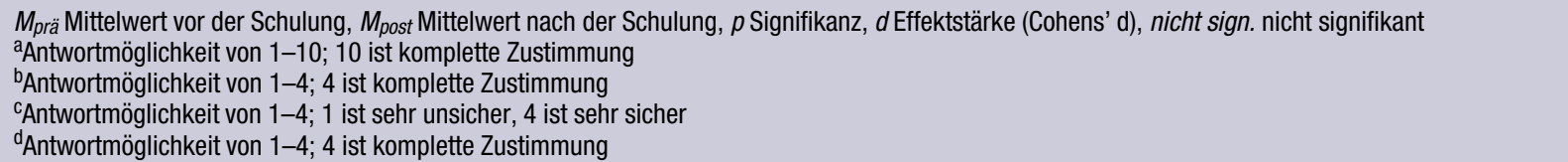 } \\
\hline
\end{tabular}

wobei die Module 1 und 2 vorwiegend auf Anfrage für ganze Stationsteams angeboten werden, während das Modul 3 für alle frei zugänglich ist. Nachdem die Versorgung von diesen Patientinnen und Patienten trotz der doch erheblichen Anzahl an Betroffenen nicht zur alltäglichen Routine zählt, ist eine regelmäßige Wiederholung der Schulungen sinnvoll und nötig.

Die Bereiche, in denen das angebotene Schulungsprogramm keine signifikanten Verbesserungen bringen konnte, beziehen sich überwiegend auf die eigene emotionale Befindlichkeit des medizinischen Fachpersonals im Umgang mit gewaltbetroffenen Patientinnen und Patienten. Unserer Ansicht nach benötigt es dafür zusätzliche Angebote, wie beispielsweise moderierte Fallbesprechungen oder auch ein spezielles Supervisionsangebot, um das Befinden in diesen Bereichen verbessern zu können. Die Notwendigkeit dieser Angebote wiederum könnte in den Schulungen dargelegt und etwaige Vorbehalte dagegen somit reduziert werden.

An den evaluierten Schulungen haben nur wenige Ärztinnen und Ärzte teilgenommen, was einerseits an strukturellen Gegebenheiten (zeitliche und personelle Ressourcen, Dienstpläne usw.) liegen kann oder dass es andererseits bisher zu wenig gelungen ist, diese Berufsgruppe zur Teilnahme an den Schulungen zu motivieren und die Relevanz des Themas auch für Ärztinnen und Ärzte deutlich zu machen. Als Konsequenz daraus könnte überlegt werden, ein spezielles Schulungsangebot für Ärztinnen und Ärzte anzubieten.

Die empfohlene Struktur der Communication Skills Trainings in der Onkologie, die in der Regel aus Basiskurs und Aufbauseminaren, Fallbesprechungen, Ver- tiefungsseminaren und Rollenspielen sowie dem Beziehungsaufbau und dem Umgang mit entstehenden Emotionen bestehen [24], lässt sich nach unseren Erfahrungen gut auf Sensibilisierungstrainings gegenüber häuslicher Gewalt übertragen. Auch wäre eine erneute Befragung der Teilnehmerinnen und Teilnehmer in einem größeren Abstand zur Schulung sinnvoll, um die Nachhaltigkeit der Ergebnisse (Gefühl der Sicherheit bei den Befragten) zu überprüfen.

Danksagung Ein großer Dank gilt allen weiteren Mitgliedern der Opferschutzgruppe des Landeskrankenhauses Innsbruck: Alexander Berger, Alexandra Ciresa-König, Christina Eisner, Nuran Ekingen, Konstantin Genelin, Daniela Gschaar-Anner, Christoph Hannemann, Matthias Haselbacher, Andrea Hohenegger, Birgit Innerhuber, Andrea Kofler, Claudia Kovac, Marion Pavlic, Adriano Perwög, Verena-Maria Schmidt, Lydia Stix, Ulrike Weber-Lau, Patrick Zechner.

Funding Open access funding provided by University of Innsbruck and Medical University of Innsbruck.

Interessenkonflikt T. Beck, I. Trawöger, D. Riedl und A. Lampe geben an, dass kein Interessenkonflikt besteht.

Open Access Dieser Artikel wird unter der Creative Commons Namensnennung 4.0 International Lizenz veröffentlicht, welche die Nutzung, Vervielfältigung, Bearbeitung, Verbreitung und Wiedergabe in jeglichem Medium und Format erlaubt, sofern Sie den/die ursprünglichen Autor(en) und die Quelle ordnungsgemäß nennen, einen Link zur Creative Commons Lizenz beifügen und angeben, ob Änderungen vorgenommen wurden.

Die in diesem Artikel enthaltenen Bilder und sonstiges Drittmaterial unterliegen ebenfalls der genannten Creative Commons Lizenz, sofern sich aus der Abbildungslegende nichts anderes ergibt. Sofern das betreffende Material nicht unter der genannten Creative Commons Lizenz steht und die be- 
treffende Handlung nicht nach gesetzlichen Vorschriften erlaubt ist, ist für die oben aufgeführten Weiterverwendungen des Materials die Einwilligung des jeweiligen Rechteinhabers einzuholen.

Weitere Details zur Lizenz entnehmen Sie bitte der Lizenzinformation auf http://creativecommons.org/licenses/by/4 $0 /$ deed.de.

\section{Literatur}

1. FRA-European Union Agency for Fundamental Rights. Violence against women: an EU-wide survey 29.2014.

2. Österreichisches Institut für Familienforschung. Gewalt in der Familie und im nahen sozialen Umfeld. Österreichische Prävalenzstudie zur Gewalt an Frauen und Männern. 2011.

3. Brzank P, Hellbernd H, Maschewsky-Schneider U, KallischniggG.HäuslicheGewaltgegen FrauenundVersorgungsbedarf. Bundesgesundheitsblatt Gesundheitsforschung Gesundheitsschutz. 2005;48:337-45.

4. Ellsberg M, Jansen H, Heise L, Watts Ch, Garcia-Moreno C. Intimatepartnerviolenceand women's physical and mental health in the WHO multi-country study on women's health and domestic violence: an observational study. Lancet. 2008;371:1165-72.

5. Campbell JC. Health consequences of intimate partner violence. Lancet. 2002;359:1331-6.

6. Sarkar NN. The impact of intimate partner violence on women's reproductive health and pregnancy outcome. Obstet Gynecol. 2008;28:266-71.

7. Campbell JA, Walker RJ, Egede LE. Associations between adverse childhood experiences, high-risk behaviors, and morbidity in adulthood. Am J Prev Med.2016;50(3):344-52.

8. Riedl D, Beck T, Exenberger S, Daniels J, Dejaco D, Lampe A. Violence from childhood to adulthood: the influence of adverse childhood experiences and domestic violence on the physical health in later life. J Psychosom Res. 2019;116:68-74.

9. Riedl D, Exenberger S, Daniels JK, Böttcher B, Beck T, Dejaco D, et al. Domestic violence victims in a hospital setting: prevalence, health impacts and patients' preferences-results from a cross-sectional study. Eur J Psychotraumatol. 2019;10:1. https://doi.org/10.1080/ 20008198.2019.1654063.

10. Krug EG, Mercy JA, Dahlberg LL, Zwi AB. The world report on violence and health. Lancet. 2008;360:1083-8.

11. Winterholler M, Wieners K. Häusliche und sexuelle Gewalt gegen Frauen. Implikationen der WHO-Leitlinien
fürDeutschland. BundesgesundheitsblattGesundheitsforschung Gesundheitsschutz. 2016;59:73-80.

12. Kothari CL, Rhodes KV. Missed opportunities: emergency department visits by police-identified victims of intimate partner violence. Ann Emerg Med. 2006;47:190-9.

13. Brzank P, Blattner B. Screening nach Gewalt gegen Frauen durch den Partner. Internationale Diskussion, ÜberlegungenfürDeutschland. BundesgesundheitsblattGesundheitsforschung Gesundheitsschutz. 2010;53:221-32.

14. Rodriguez MA, Bauer HM, McLoughlin E, Grumbach K. Screening and intervention for intimate partner abuse: practices and attitudes of primary care physicians. JAMA. 1999;282:468-74.

15. Rodriguez MA, Quiroga SS, Bauer HM. Breaking the silence: battered women's perspectives on medical care. Arch Fam Med. 1996;5:153-8.

16. Wasson JH, Jette AM, Anderson J, Johnson DJ, Nelson EC, Kilo CM. Routine, single-item screening to identify abusive relationships in women. J Fam Pract. 2000;49:1017-22.

17. Ramsay J, Richardson J, Carter YH, Davidson LL, Feder G. Should health professionals screen women for domestic violence? Systematic review. BMJ. 2002;324:314-27.

18. Nyberg E, Hartmann P, Stieglitz R, Riecher-Rössler A. Screening Partnergewalt: Ein deutschsprachiges Screeninginstrument für häusliche Gewalt gegen Frauen. Fortschr Neurol Psychiatr. 2008;76:28-36.

19. Nicolaidis C. The voices of survivors documentary. Using patient narrative to educate physicians about domestic violence.J Gen Intern Med. 2002;17:117-24.

20. RepublikÖsterreich (2011), Bundesgesetzblatt 69 .

21. Graß H, Berendes L, Mützel E, Preuss R, Ritz-Timme S. Medizinische Intervention gegen Gewalt an Frauen. Rechtsmedizin. 2013;23:180-5.

22. Cohen J. Statistical power analysis for the behavioral sciences. 2. Aufl. NewYork: Erlbaum; 1988.

23. Ellis PD. The essential guide to effect sizes: statistical power, meta-analysis, and the interpretation of research results. Cambridge, NewYork: Cambridge University Press; 2010.

24. Stiefel F, Barth J, Bensing J, Fallowfield L, Jost L, Razavi D, et al. Communication skills training in oncology: A position paper based on a consensus meeting among European experts in 2009. Ann Oncol.2010;21:204-7.

Hinweis des Verlags Der Verlag bleibt in Hinblick auf geografische Zuordnungen und Gebietsbezeichnungen in veröffentlichten Karten und Institutsadressen neutral. 